\begin{tabular}{|c|c|}
\hline Title & Retrospective and prospective information coding by different neurons in the prefrontal cortex \\
\hline Author(s) & Matsushima, A y ano; T anaka, Masaki \\
\hline Citation & $\begin{array}{l}\text { Neuroreport, 24(2), } 73.78 \\
\text { https://doi.org/10.1097/WNR.0b013e32835c8d66 }\end{array}$ \\
\hline Issue Date & 2013-01-23 \\
\hline Doc URL & http:/hdl.handle.net/2115/57881 \\
\hline Rights & $\begin{array}{l}\text { This is a non-final version of an article published in final form in [Matsushima, Ayano; Tanaka, Masaki. Retrospective } \\
\text { and prospective information coding by different neurons in the prefrontal cortex. Neuroreport. 24(2):73-78, January 23, } \\
\text { 2013]. }\end{array}$ \\
\hline Type & article (author version) \\
\hline File Information & NeuroReport2013(HUSCUP).pdf \\
\hline
\end{tabular}

Instructions for use 


\section{Retrospective and prospective information coding by different neurons in the prefrontal cortex}

Ayano Matsushima \& Masaki Tanaka

Department of Physiology, Hokkaido University School of Medicine, Sapporo 060-8638, Japan

Running head: Separate representations of past and future

Abstract: 249 words

Text pages: 14

References: 25

Figures: 3

Supplemental digital content: 1.pdf

The authors declare no conflict of interest.

Supported by the MEXT and MHLW of Japan; the Takeda foundation; the Uehara Memorial Foundation; the Brain Science Foundation; the Smoking Research Foundation; the Akiyama Life Science Foundation; and the Suhara Memorial Foundation. A.M. was a Graduate Research Fellow of the JSPS. Animals were provided by the NBRP.

Correspondence to:

Dr. Masaki Tanaka

Department of Physiology

Hokkaido University School of Medicine

North 15, West 7, Sapporo 060-8638, Japan

Tel: +81 11-706-5039, Fax: +81 11-706-5041

E-mail: masaki@med.hokudai.ac.jp 


\section{Abstract}

Neurons in the lateral prefrontal cortex exhibit sustained activity during the maintenance of visual memory. Previous studies also demonstrated that prefrontal neurons display predictive activity in anticipation of upcoming visual stimuli. Because these retrospective and prospective coding of visual stimuli have been examined in separate experiments, how these processes interact in individual neurons remains unknown. To examine this, we recorded from single prefrontal neurons while monkeys performed two behavioural tasks. In one task, the animals passively viewed a moving object during fixation, while in the other, they remembered the location of a briefly presented visual cue for subsequent saccades. We found that many neurons were reactive and responded only after the visual stimulus appeared in their receptive field, whereas some neurons were predictive and increased their activity even before the moving stimulus entered the receptive field. In the memory-guided saccade trials, the reactive neurons exhibited sustained activity during the delay period, whereas the predictive neurons did not. Delays of visual response to a moving stimulus did not correlate with visual latency for a stationary stimulus. Instead, it correlated with the magnitude of sustained activity during the delay period in the memory-guided saccade task. Our data show that retrospective and prospective coding of visual information are represented by distinct neuronal populations, and that their temporal preferences are stable across different task conditions. Reactive signals may reflect the amount of temporal integration in short-term memory, whereas predictive signals may solely represent future events in isolation from the maintenance of past information.

Key words: prefrontal cortex, working memory, prediction, single neurons, primate 


\section{Introduction}

It has been widely accepted that the lateral prefrontal cortex is essential for working memory. Performance in tasks requiring short-term memory is known to be impaired in subjects with prefrontal lesions [1,2], and regional blood flow in the prefrontal cortex increases when subjects temporally store previous information for subsequent actions $[3,4]$. The neuronal basis underlying working memory has also been examined in monkeys using a variety of delayed response tasks [5-7]. For example, neurons in the dorsolateral prefrontal cortex exhibit sustained activity when animals remember the location of a previously presented visual stimulus [8,9]. Because the magnitude of neuronal activity is spatially tuned, these neurons are believed to serve as a mnemonic code for stimulus location.

In addition to retention of past information, the lateral prefrontal cortex is also involved in predicting future events. For example, a focal prefrontal lesion results in severe impairments in the generation of anticipatory eye movements [10-12], and schizophrenic patients exhibit difficulty in motion prediction tasks [13]. Neuronal activities in the prefrontal cortex are modulated by the direction of forthcoming actions [14] or visual events triggering movements [15]. Other studies have shown predictive shifts of the visual receptive field in advance of saccades [16,17] or during the covert tracking of invisible objects [18], as well as a gradual rise of neuronal activity in anticipation of target presentation in the receptive field [19]. All of these observations indicate that the prefrontal cortex is capable of predicting future events so as to compensate for neural delays imposed on sensory processing.

Although both the memory- and prediction-related signals in the prefrontal cortex have been well documented, little is known about how these signals interact with 
each other. In particular, sustained and predictive visual responses in the prefrontal cortex, which could be viewed as retrospective and prospective representations of visual stimuli, have been examined separately. In this study, we employed two behavioural paradigms in an attempt to elucidate possible interaction between these signals in individual neurons.

\section{Materials and Methods}

Animal preparation

Two female Japanese monkeys (Macaca fuscata, 6-7 kg) were used. All experimental protocols were approved in advance by the Animal Care and Use Committee of Hokkaido University. The procedures used for animal preparation are described elsewhere in detail $[20,21]$. Briefly, a pair of head holders was implanted on the skull using titanium screws and dental acrylic under general isoflurane anaesthesia. A coil of stainless steel wire was implanted under the conjunctiva to record eye movements. During subsequent training and experimental sessions, the monkey's head was secured to the primate chair, and eye movements were recorded using the search coil technique (MEL-25; Enzanshi Kogyo). After training on behavioural tasks, a recording cylinder was installed over a small craniotomy, allowing electrode penetrations into the prearcuate prefrontal cortex (Fig. 1b). Animals received analgesia after each surgery. The monkeys' water intake was controlled daily so that they were motivated to perform the tasks.

Visual stimuli and behavioural paradigms

All experiments were controlled by a Windows-based real-time data acquisition system 
(TEMPO; Reflective Computing). Visual stimuli were presented on a 24-inch cathode-ray tube monitor (GDM-FW900; Sony) positioned $38 \mathrm{~cm}$ from the eyes, subtending $64 \times 44^{\circ}$ of the visual angle. All visual stimuli were presented within a $40^{\circ}$ square contour that was visible throughout the experiments (square boundaries in Fig. 1a). Animals were trained on the covert tracking, memory-guided saccade, and smooth pursuit tasks [20]. In the present study, we examined neuronal activity during the single target mode of the covert tracking task and during the memory-guided saccade trials.

In the single target mode of the covert tracking trials (Fig. 1a), a white circle $\left(2^{\circ}\right)$ was presented at $10^{\circ}$ eccentricity, at one of 36 polar angles $\left(0-350^{\circ}, 10^{\circ}\right.$ increments), during central fixation. Colour of the circle altered briefly to red (cue period, $300 \mathrm{~ms}$ ), and a 300-ms delay period followed. Then, the stimulus moved along straight paths at $20 \% \mathrm{~s}$ in different directions, and bounced against the sides of the $40^{\circ}$ square (motion period, $3000 \mathrm{~ms}$ ). The initial motion direction was chosen randomly between $0^{\circ}$ and $330^{\circ}$ ( $30^{\circ}$ steps). Monkeys were required to keep their eyes within $5^{\circ}$ of the fixation point throughout the motion and the following delay (1000 ms) periods, then to make a saccade to the stimulus within $400 \mathrm{~ms}$ after the fixation point offset.

In the memory-guided saccade trials, a visual cue $\left(1^{\circ}\right.$ square $)$ was presented briefly (200 ms) at $12^{\circ}$ eccentricity during central fixation. Monkeys were required to remember its location and maintained fixation for an additional 2000 ms. Once the fixation point disappeared, the animals were required to make a saccade to the previously cued location within 400 ms.

\section{Physiological procedures}

Neuronal activity was recorded through tungsten electrodes (FHC lnc. or Alpha Omega Engineering). The location of the electrode penetration was adjusted using the $\mathrm{X}-\mathrm{Y}$ 
stage attached to the cylinder (MO-97S; Narishige). Neuronal signals were amplified (Model 1800; A-M Systems), filtered (Model 3625; NF Co.), and monitored online using oscilloscopes and an audio device. The waveforms of a single neuron were isolated using a real-time spike sorter with template-matching algorithms (MSD; Alpha Omega Engineering). Occurrences of action potentials were time stamped and saved in files with the data for eye movements and stimulus locations during experiments (sampling rate: $1 \mathrm{kHz}$ ).

\section{Data analysis}

The data were analysed offline using Matlab (Mathworks). We considered only neurons exhibiting a significant firing modulation depending on the stimulus location during the motion period in the covert tracking task, as in the previous study [20]. Briefly, the instantaneous firing rate was calculated for each stimulus location, which was filtered with a 2-D Gaussian kernel $\left(\sigma=2.5^{\circ}, 20^{\circ}\right.$ square, $0.5^{\circ}$ resolution $)$ to obtain a response map. The magnitude of firing modulation was quantified by the variance of firing rates in the response map. To evaluate the significance level, the correspondence between the spike data and the stimulus trajectories was shuffled 1000 times to obtain the null distribution. Neurons exhibiting a greater variance of firing rates than expected by chance $(P<0.05)$ were included for further analyses (79\%, 245/311).

To measure the delay in neuronal response to the moving stimulus, we first constructed a response map for each time shift $(\Delta \mathrm{t})$ ranging from -700 to $700 \mathrm{~ms}$ (20 ms steps), with a positive value indicating neuronal activity lagged behind the stimulus. For this analysis, a broader Gaussian kernel $\left(\sigma=4^{\circ}, 20^{\circ}\right.$ square, $1^{\circ}$ resolution) was used to filter out any incidental activity. The peak firing rate was plotted as a function of $\Delta \mathrm{t}$ (Fig. 1d), and then the data were fitted with a Gaussian centred within $\pm 400 \mathrm{~ms}$. If the fitted 
curve explained most of the variance $\left(r^{2} \geq 0.75,61 \%, 150 / 245\right)$ and the locations of the receptive field centre evaluated by a bootstrap procedure were stable (1000 repeats, SD $\left.\leq 3^{\circ}, 57 \%, 85 / 150\right)$, the mean of the fitted Gaussian was taken as the optimal delay for a given neuron.

For the memory-guided saccade task, firing rates were measured during a 300-ms interval immediately before the cue onset (baseline), a 200-ms interval starting from 50 ms after the cue onset (cue period), a 400-ms interval just before the fixation point offset (delay period), and a 200-ms interval centred at saccade initiation (saccade period). The firing rates during these periods were compared using a one-way ANOVA. Neurons were classified as having cue, delay, or saccade responses when post hoc multiple comparisons (Scheffé's test) revealed significant changes in firing during the corresponding intervals compared with baseline activity $(P<0.05$, Fig. 3b). The magnitude of modulation in each period was quantified by computing the modulation index (MI), defined as MI = (Resp - Baseline $) /($ Resp + Baseline $)$, where Resp indicates the firing rate during either the cue, delay, or saccade period (Fig. 3c).

To measure the visual response latency in the memory-guided saccade task, the activities for the cue presented in the receptive field were compared with those for the cue in the opposite direction. The receiver operating characteristics (ROC) value was computed for every $1 \mathrm{~ms}$, and the first of 5 consecutive time windows with ROC values $>0.8$ was defined as the onset of visual response.

\section{Results}

We recorded from single neurons in the lateral prefrontal cortex, mostly in area 8 (Fig. 1b) $[22,23]$, during monkeys passively viewed a moving object (Fig. 1a). An example 
neuron shown in Figure 1c had a receptive field in the right visual field, exhibiting the greatest firing modulation for the delay of $41 \mathrm{~ms}$ (Fig. 1d). Figure 1e summarises the optimal delay measured for 85 neurons, showing that activities of 28 neurons (33\%) preceded, whereas those of 57 neurons (67\%) followed the stimulus presentation in the receptive field. Considering the delay for visual processing, the 85 neurons were divided into the reactive ( $n=42)$ and predictive $(n=43)$ neuron groups at their median value of 47 ms (Fig. 1e), which was in agreement with the visual response latencies in FEF in the literature [24]. In our previous study, we used the covert tracking task with a distractor and found that a group of prefrontal neurons responded more to the distractor than to the target, whereas the others responded more to the target than to the distractor. The object preference of each neuron was constant across a wide range of $\Delta \mathrm{t}$ (Supplemental Digital Content 1), indicating that the object selectivity reported previously reflected an intrinsic property of the neuron rather than an artefact for a specific $\Delta t$.

Assuming that the delay of visual response for each neuron is constant, the response field measured for the stimuli approaching or moving away from the receptive field might exhibit an apparent shift. To examine this, the mean of the instantaneous firing rates were computed for every combination of stimulus speed and location relative to the centre of the receptive field (every $2 \%$ s and $2^{\circ}$, Fig. 2). For example, the bottom row of each panel in Figure 2 plots neuronal firing as the stimulus moved toward or away from the centre of the receptive field at a component speed of $18-20 \%$ s. The centre of mass calculated for each speed shifted horizontally (blue dots) for both types of neurons. The shift of the response field was in the direction of stimulus motion (a) or in the opposite direction (b), indicating that the reactive neurons tended to respond to the stimulus moving away from the receptive field, whereas the predictive 
neurons tended to respond to the approaching stimulus. The magnitude of the shift significantly correlated with the stimulus speed for both types of neurons ( $r=0.98$ and $-0.90, P<0.0001)$.

We next examined whether the reactive and predictive neurons exhibited different patterns of activity during the memory-guided saccade trials. Among 51 neurons tested, 45 (24 reactive, 21 predictive neurons) exhibited at least one of the cue, delay, or saccade-related responses (Fig. 3b, see Methods). Figure 3a plots the time courses of population activity for both types of neurons, showing greater activity throughout the delay and the saccade periods for the reactive neurons than the predictive neurons. For individual neurons, the optimal delay correlated with the modulation index computed for the delay period ( $P<0.01$, Fig. 3c) but not for the other two task periods. The visual response latency for the cue in the memory-guided saccade trials and the optimal delay measured in the tracking trials were not correlated with each other $(r=$ $0.27, P>0.1, n=29)$. These results suggest that the visual delay for a continuously moving object may be determined by the sustained visual response rather than the latency or magnitude of transient visual responses.

\section{Discussion}

The present study is the first to directly compare the temporal properties of visual responses to a moving object with the functional properties during a spatial working memory task in individual neurons in the lateral prefrontal cortex. We found two types of neurons. One group responded to the moving stimulus with a delay that was shorter than visual latency measured for a stationary object. When neuronal activity was further examined for each combination of object speed and location relative to the centre of the 
receptive field, we found that those neurons responded strongly to a stimulus that was approaching but had not reached the centre of the receptive field (Fig. 2b). A similar shift of response field in the direction opposite to the object motions has also been reported in V4 neurons, as monkeys passively view apparent motion stimuli [25]. The finding of predictive signals during passive viewing in this and previous studies suggests that the brain may automatically extrapolate and expect the future location of a moving object when its trajectory is readily predictable. Importantly, many of these neurons exhibited decreased activity during the delay period in the memory-guided saccade task (Fig, 3a), suggesting that they may be involved in prospective rather than retrospective coding of visual information.

The other group of neurons responded to a moving object with a longer delay. The preferred stimulus location tended to be shifted in the direction of object motion, and the amount of shift linearly correlated with object speed (Fig. 2a). Because these neurons were active during the delay period in the memory-guided saccade trials, they appeared to represent visual memory and integrate signals over time to retrospectively encode spatial information. Counterintuitively, the visual delay for the moving object correlated with neither the latency nor the magnitude of visual response measured in the memory-guided saccade trials. Instead, the temporal properties of the visual response to a moving stimulus were linked with the functional properties for working memory in single prefrontal neurons (Fig. 3c). Our results suggest that reactive and predictive visual information in the lateral prefrontal cortex are processed by two distinct neuronal populations, which might reflect different amounts of temporal integration for visual inputs. 


\section{Acknowledgements}

We thank T. Shiraishi and T. Mori for animal care, and M. Suzuki for administrative help. 


\section{References}

1 Bauer RH, Fuster JM. Delayed-matching and delayed-response deficit from cooling dorsolateral prefrontal cortex in monkeys. J Comp Physiol Psychol 1976; 90: 293-302.

2 Kojima S, Kojima M, Goldman-Rakic PS. Operant behavioral analysis of memory loss in monkeys with prefrontal lesions. Brain Res 1982; 248: 51-59.

3 Brown MR, DeSouza JF, Goltz HC, Ford K, Menon RS, Goodale MA et al. Comparison of memory- and visually guided saccades using event-related fMRI. J Neurophysiol 2004; 91: 873-889.

4 Cabeza R, Nyberg L. Imaging cognition II: An empirical review of 275 PET and fMRI studies. J Cogn Neurosci 2000; 12: 1-47.

5 Fuster JM, Alexander GE. Neuron activity related to short-term memory. Science 1971; 173: 652-654.

6 Kojima S, Goldman-Rakic PS. Delay-related activity of prefrontal neurons in rhesus monkeys performing delayed response. Brain Res 1982; 248: 43-49.

7 Kubota K, Niki H. Prefrontal cortical unit activity and delayed alternation performance in monkeys. J Neurophysiol 1971; 34: 337-347.

8 Funahashi S, Bruce CJ, Goldman-Rakic PS. Mnemonic coding of visual space in the monkey's dorsolateral prefrontal cortex. J Neurophysiol 1989; 61: 331-349.

9 Constantinidis C, Franowicz MN, Goldman-Rakic PS. The sensory nature of mnemonic representation in the primate prefrontal cortex. Nat Neurosci 2001; 4: 311-316.

10 Heide W, Kurzidim K, Kompf D. Deficits of smooth pursuit eye movements after frontal and parietal lesions. Brain 1996; 119: 1951-1969.

11 Keating EG. Frontal eye field lesions impair predictive and visually-guided pursuit eye movements. Exp Brain Res 1991; 86: 311-323.

12 MacAvoy MG, Gottlieb JP, Bruce CJ. Smooth-pursuit eye movement representation in the primate frontal eye field. Cereb Cortex 1991; 1: 95-102.

13 Hooker C, Park S. Trajectory estimation in schizophrenia. Schizophr Res 2000; 45: 83-92.

14 Watanabe Y, Takeda K, Funahashi S. Population vector analysis of primate mediodorsal thalamic activity during oculomotor delayed-response performance. Cereb Cortex 2009; 19: 1313-1321.

15 Lebedev MA, Messinger A, Kralik JD, Wise SP. Representation of attended versus remembered locations in prefrontal cortex. PLoS Biol 2004; 2: e365.

16 Sommer MA, Wurtz RH. Influence of the thalamus on spatial visual processing in frontal cortex. Nature 2006; 444: 374-377.

17 Umeno MM, Goldberg ME. Spatial processing in the monkey frontal eye field. I. Predictive visual responses. J Neurophysiol 1997; 78: 1373-1383.

18 Xiao Q, Barborica A, Ferrera VP. Modulation of visual responses in macaque frontal eye field during covert tracking of invisible targets. Cereb Cortex 2007; 17:918-928.

19 Zhou HH, Thompson KG. Cognitively directed spatial selection in the frontal eye field in anticipation of visual stimuli to be discriminated. Vision Res 2009; 49: 1205-1215. 
20 Matsushima A, Tanaka M. Neuronal correlates of multiple top-down signals during covert tracking of moving objects in macaque prefrontal cortex. J Cogn Neurosci 2012; 24: 2043-2056.

21 Tanaka M. Involvement of the central thalamus in the control of smooth pursuit eye movements. J Neurosci 2005; 25: 5866-5876.

22 Petrides M, Pandya DN. Comparative cytoarchitectonic analysis of the human and the macaque ventrolateral prefrontal cortex and corticocortical connection patterns in the monkey. Eur J Neurosci 2002; 16: 291-310.

23 Petrides MC. Lateral prefrontal cortex: architectonic and functional organization. Philos Trans R Soc Lond B Biol Sci 2005; 360: 781-795.

24 Pouget P, Emeric EE, Stuphorn V, Reis K, Schall JD. Chronometry of visual responses in frontal eye field, supplementary eye field, and anterior cingulate cortex. $J$ Neurophysiol 2005; 94: 2086-2092.

25 Sundberg KA, Fallah M, Reynolds JH. A motion-dependent distortion of retinotopy in area V4. Neuron 2006; 49: 447-457. 


\section{Figure legends}

Fig. 1 (a) Sequence of events in the single target mode of the covert tracking paradigm. (b) Locations of the recording sites. AS; arcuate sulcus. PS; principal sulcus. (c) Responses of a representative neuron. Instantaneous firing rates as a function of object locations were computed for different delays $(\Delta t)$. (d) The peak firing rate measured for each response map as a function of $\Delta \mathrm{t}$. Error bars indicate SEM (bootstrap SD). The data were well fitted with a Gaussian centred at $41 \mathrm{~ms}$ (red broken curve, $r^{2}=0.97$ ). (e) Distribution of the optimal delay for 85 neurons.

Fig. 2 Reactive and predictive responses to a moving object. Instantaneous firing rates for a variety of combinations of object speed and distance from the centre of the receptive field, computed for the reactive (a) and predictive (b) neurons. Blue dots indicate the average object locations weighted by the firing rates in each row.

Fig. 3 Different response properties of two types of neurons during memory-guided saccades. (a) Time courses of population activity for the reactive (red) and predictive (blue) neurons in trials presented in the receptive field. Top green dots indicate a significant difference between the traces (running $T$ test for every millisecond, $P<0.05$ ). Shaded areas indicate 95\% confidence intervals. (b) Proportions of reactive (red bar) and predictive (blue bar) neurons exhibiting cue, delay, and saccade-related responses. Unfilled bars indicate neurons with inhibitory responses. (c) Relationship between the modulation indices and the optimal delay. Solid black lines indicate running averages of 10 consecutive data points along the horizontal axis.

Supplemental Digital Content 1.pdf 


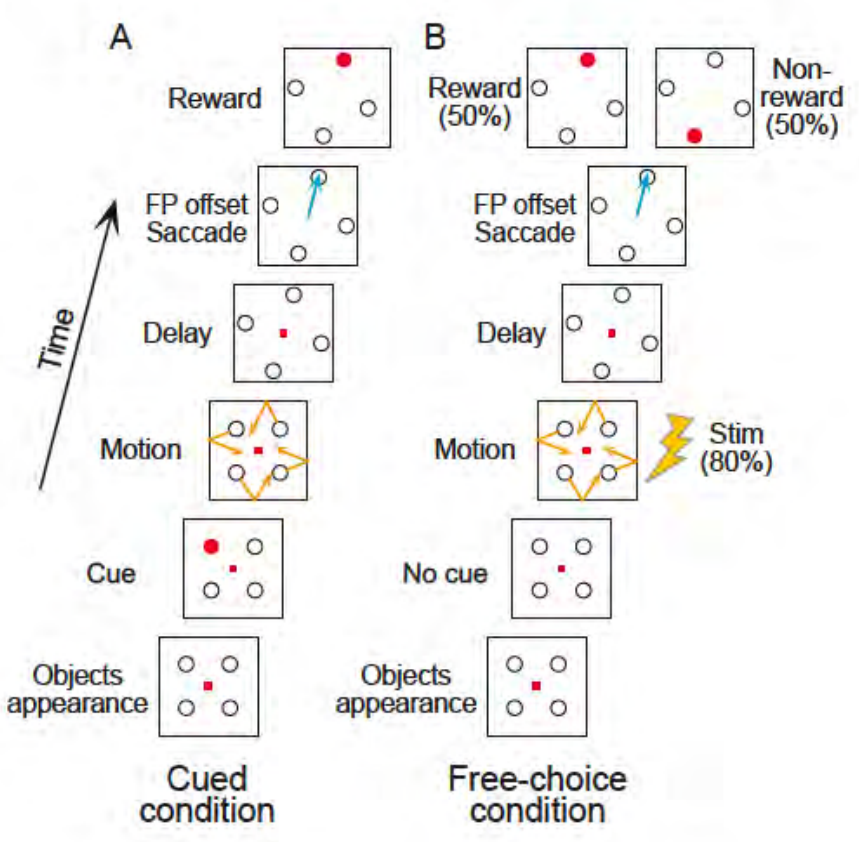

Figure 1, Matsushima \& Tanaka 

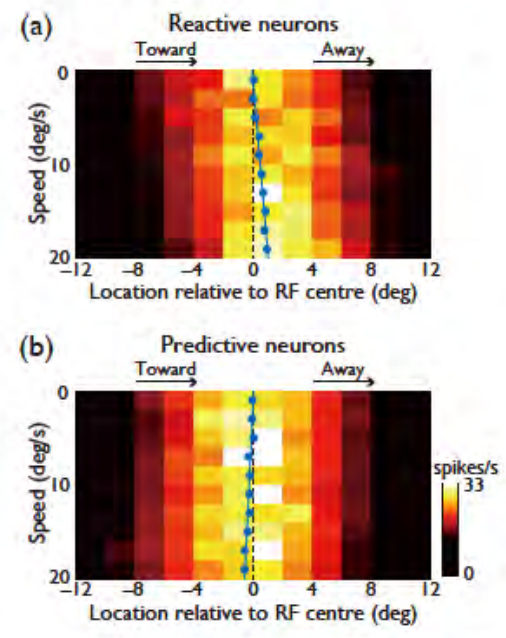

Figure 2, Matsushima \& Tanaka 

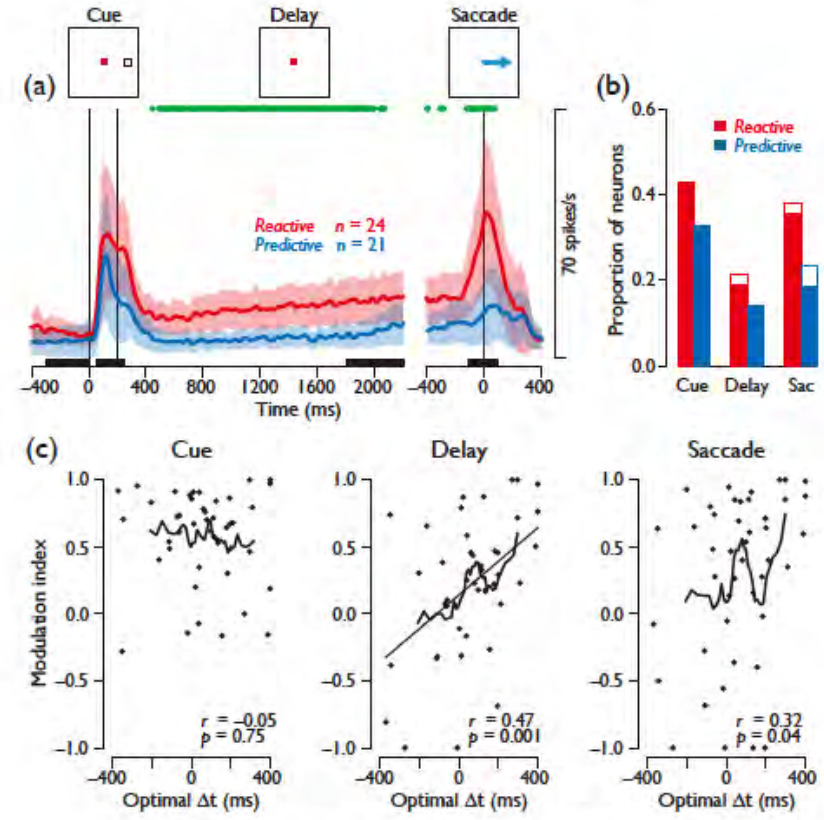

Figure 3, Matsushima \& Tanaka 


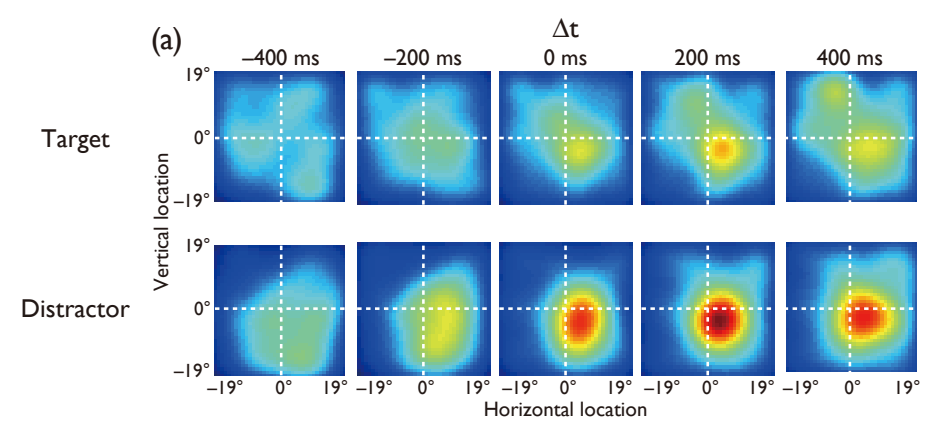

(b)
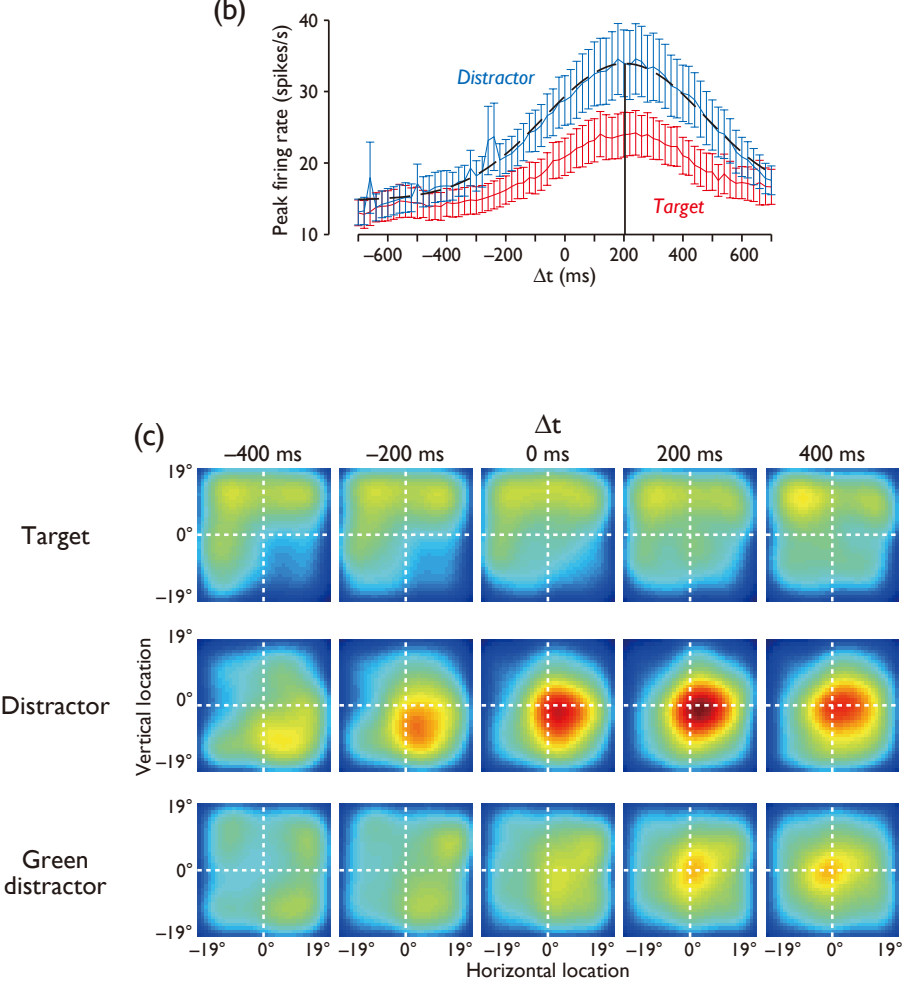

(d)

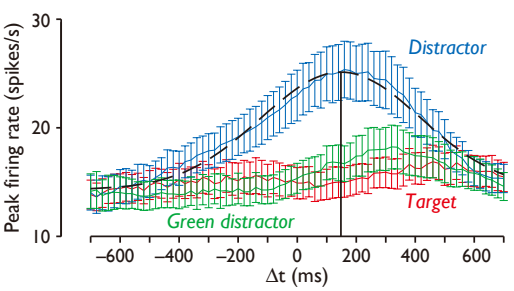

Fig. S1 The optimal delay measured for distractor-selective neurons. (a) Instantaneous firing rates as a function of target (upper row) and distractor (lower row) locations were computed for different delays $(\Delta t)$. (b) Peaks in the response maps as a function of $\Delta t$. (c and d) The response of another distractor-selective neuron examined in the modified covert tracking task with a green distractor. Note that the response to the distractor was greater than that to the other objects, irrespective of the $\Delta t$ used to compute the response maps. The data from these neurons were presented in our previous paper (Figs. 5 and 8 of Matsushima and Tanaka, 2012). Among 85 neurons tested in the present study, $71(84 \%)$ and $9(11 \%)$ exhibited target-selective and distractor-selective responses, respectively. The optimal delays measured in the single target trials were not significantly different between the two types of neurons $(61 \pm 193 \mathrm{~ms}$, median: $59 \mathrm{~ms}$ vs. $-78 \pm 184 \mathrm{~ms}$, median: $154 \mathrm{~ms}$; Wilcoxon rank-sum test, $\mathrm{P}>0.7)$. 